\title{
Parents may exert a greater influence on children's food and nutrient intakes than the school environment in 9-10 year olds
}

\author{
A. Jennings ${ }^{1}$, A. Welch ${ }^{1}$, I. Harvey ${ }^{1}$, P. Skidmore ${ }^{1}$, K. Le Cornu ${ }^{1}$, E. van Sluijs ${ }^{3}$, F. Harrison ${ }^{2}$, \\ S. Griffin ${ }^{3}$ and A. Cassidy ${ }^{1}$ \\ ${ }^{1}$ School of Medicine and ${ }^{2}$ School of Environmental Sciences, University of East Anglia, Norwich NR4 7TJ, UK \\ and ${ }^{3}$ MRC Epidemiology Unit, Addenbrookes Hospital, Cambridge CB2 OQQ, UK
}

The diets of schoolchildren are a key health issue in the UK. Public health interventions to improve diet need to be evidence based but, to date, relatively little is known about the key determinants of food choice and in particular the relative influence of school and parents.

A population-based sample of 1859 children ( 815 boys and 1044 girls, $57 \%$ response rate, $90 \%$ for diary completion) from ninety-two schools in Norfolk, England completed the Sport, Physical activity and Eating behaviour: Environmental Determinants in Young people (SPEEDY) study ${ }^{(1)}$. Children's diet was assessed using $4 \mathrm{~d}$ diaries, data on parental fruit and vegetable intake were collected by questionnaire and school policies and approaches to improved healthy eating were used to derive a 'healthy school' classification. The healthiest schools provided cookery lessons, extra-curricular food-related activities, information on healthy eating beyond the national curriculum, a policy to promote healthy eating and regulations on the food that children could consume at break time and that was sold in the school. The associations between parental diet and the school environment and children's food and nutrient intakes were examined using analysis of covariance and adjusting for parental education and gender (SPSS version 14.0; SPSS Inc., Chicago, IL, USA) and the percentage difference in children's dietary intake between the highest and lowest categories of parental fruit and vegetable consumption and highest and lowest categories of school classification was calculated.

Both parental diet and school were associated with food and nutrient intake in children but the association of parental diet with fruit and vegetable intake was twice the scale of the school (one portion $v .0 .5$ portions). Specifically, comparing parents in the highest $v$ lowest categories of fruit and vegetable intake there was a $23.1 \%$ difference in children's fruit and vegetable intake ( $41 \mathrm{~g}$, equivalent to one portion), a $28.2 \%$ difference in vitamin C, a $4.7 \%$ difference in NSP and a $-4.8 \%$ difference in saturated fat. Similar but smaller differences were found with increasing 'healthy school' index; $11.6 \%$ for fruit and vegetables, $4.2 \%$ for vitamin C, $2.9 \%$ for NSP and $-0.7 \%$ for saturated fat.

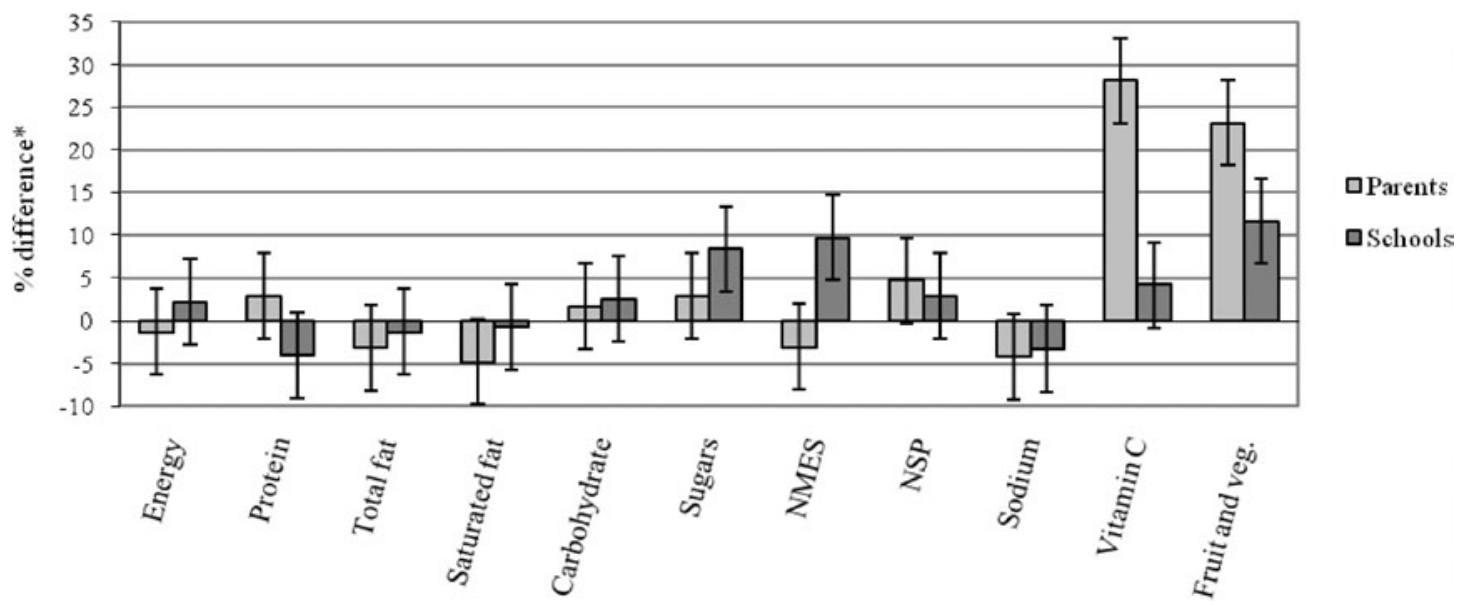

*Percentage difference was calculated as intake in the highest classification (parents, more than six portions; schools, six to seven points) minus the intakes in the lowest classification (parents, none to two portions; schools, none to two points) divided by the lowest classification and multiplied by 100 .

This study found that associations with children's dietary intakes were greatest for parental intake; however, the impact of the school environment was also evident. This is one of the first UK studies to examine the association between both parental diet and the school environment and food and nutrient intake in children. It has shown that whilst it remains important to continue to target schools to improve children's eating patterns there is also evidence that strongly suggests targeting interventions at a family level in this age-group.

1. van Sluijs E, Skidmore P, Mwanza K et al. (2008) BMC Public Health 8, 388. 